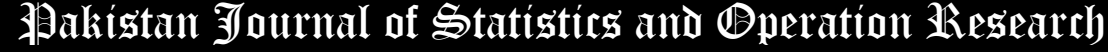

\section{Odd Generalized N-H Generated Family of Distributions with Application to Exponential Model}

\author{
Zubair Ahmad $^{1 *}$, M. Elgarhy ${ }^{2}$, G.G. Hamedani ${ }^{3}$, Nadeem Shafique Butt $^{4}$
}

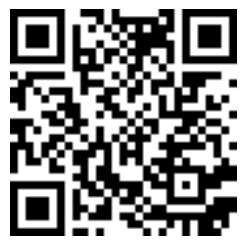

\author{
Corresponding Author \\ 1. Department of Statistics, Yazd University, P.O. Box 89175-741, Yazd, Iran, zubair@stu.yazd.ac.ir, \\ z.ferry21@gmail.com \\ 2. Valley High Institute for Management Finance and Information Systems, Obour, Qaliubia 11828, Egypt, \\ m_elgarhy85@yahoo.com \\ 3. Department of Mathematical and Statistical Sciences Marquette University Milwaukee, \\ WI 53201-1881, USA, g.hamedani@mu.edu \\ 4. Department of Family and Community Medicine, King Abdulaziz University, KSA, nshafique@kau.edu.sa
}

\begin{abstract}
A new family of distributions called the odd generalized N-H is introduced and studied. Four new special models are presented. Some mathematical properties of the odd generalized N-H family are studied. Explicit expressions for the moments, probability weighted, quantile function, mean deviation, order statistics and Rényi entropy are investigated. Characterizations based on the truncated moments, hazard function and conditional expectations are presented for the generated family. Parameter estimates of the family are obtained based on maximum likelihood procedure. Two real data sets are employed to show the usefulness of the new family.
\end{abstract}

Key Words: N-H distribution; Order statistics; Maximum likelihood method.

\section{Introduction}

In recent years, several classes have been defined by adding one or more parameters to generate new distributions. These distributions extend well-known distributions as well as provide great flexibility to model specific real data. Some of the well-known generators are the beta-G by Eugene et al. (2002), Kumaraswamy-G by Cordeiro and de Castro (2011), exponentiated generalized-G by Cordeiro et al. (2013), Transformed-Transformer (T-X) by Alzaatreh et al. (2013), Weibull-G by Bourguignon et al. (2014), exponentiated half-logistic-G by Cordeiro et al. (2014), odd generalized exponential by Tahir (2015), beta odd log-logistic generalized by Cordeiro et al. (2016), Garhy-G by Elgarhy et al. (2016), exponentiated Weibull-G by Hassan and Elgarhy (2016a), Kumaraswamy Weibull-G by Hassan and Elgarhy (2016b), exponentiated extended-G by Elgarhy et al. (2017), type II half logistic-G by Hassan et al. (2017), odd Burr generalized by Alizadeh et al. (2017), generalized odd log-logistic by Cordeiro et al. (2017), a new generalized odd log-logistic by Haghbin et al. (2017), odd Lindley-G by Gomes-Silva (2017), among others.

In this paper, we introduce a new generated family of distributions using the NH distribution as a generator. The NH distribution is introduced by (Nadarajah and Haghighi, 2011) which has the following cumulative distribution function (cdf)

$$
G(t)=1-e^{\left\{1-(1+\lambda t)^{\alpha}\right\}}=1-C e^{-(1+\lambda t)^{\alpha}}, t>0, \lambda, \alpha>0
$$


where $C=e$. The associated probability density function (pdf) corresponding to (1) is as follows

$$
g(t)=\alpha \lambda C(1+\lambda t)^{\alpha-1} e^{-(1+\lambda t)^{\alpha}},
$$

where $\lambda$ is the scale parameter and $\alpha$ is the shape parameter. We are interested in modeling the random variable $X$ of this odds using the NH model (with scale parameter $\lambda=1$ ) given by (1). The cdf of OGNH-G family can be expressed as follows

$$
F(x)=\int_{0}^{\left(\frac{G(x ; \zeta)}{1-G(x ; \zeta)}\right)^{\beta}} C \alpha(1+t)^{\alpha-1} e^{-(1+t)^{\alpha}} d t=1-C e^{-\left(1+\left(\frac{G(x ; \zeta)}{1-G(x ; \zeta)}\right)^{\beta}\right)^{\alpha}}, x \in \mathbb{R}, \alpha, \beta>0,
$$

where, $\alpha, \beta$ are two shape parameters, and $G(x ; \zeta)$ is a baseline cdf, which depends on a parameter vector $\zeta$. The distribution function (3) provides a broadly odd half logistic generated distributions. Therefore, the pdf of the OGNH$\mathrm{G}$ family is as follows

$$
f(x)=\alpha \beta C g(x ; \zeta) \frac{G(x ; \zeta)^{\beta-1}}{(1-G(x ; \zeta))^{\beta+1}}\left(1+\left(\frac{G(x ; \zeta)}{1-G(x ; \zeta)}\right)^{\beta}\right)^{\alpha-1} e^{-\left(1+\left(\frac{G(x ; \zeta)}{1-G(x ; \zeta)}\right)^{\beta}\right)^{\alpha}} .
$$

Hereafter, a random variable $X$ has pdf (4) will be denoted by $X \sim O G N H-G$.

The survival function, hazard rate and reversed hazard rate functions are, respectively, given by

$$
\begin{gathered}
\bar{F}(x)=C e^{-\left(1+\left(\frac{G(x ; \zeta)}{1-G(x ; \zeta)}\right)^{\beta}\right)^{\alpha}}, \\
h(x)=\alpha \beta g(x ; \zeta) \frac{G(x ; \zeta)^{\beta-1}}{(1-G(x ; \zeta))^{\beta+1}}\left(1+\left(\frac{G(x ; \zeta)}{1-G(x ; \zeta)}\right)^{\beta}\right)^{\alpha-1}
\end{gathered}
$$

and

$$
\tau(x)=\frac{\alpha \beta C g(x ; \zeta) \frac{G(x ; \zeta)^{\beta-1}}{(1-G(x ; \zeta))^{\beta+1}}\left(1+\left(\frac{G(x ; \zeta)}{1-G(x ; \zeta)}\right)^{\beta}\right)^{\alpha-1} e^{-\left(1+\left(\frac{G(x ; \zeta)}{1-G(x ; \zeta)}\right)^{\beta}\right)^{\alpha}}}{1-C e^{-\left(1+\left(\frac{G(x ; \zeta)}{1-G(x ; \zeta)}\right)^{\beta}\right)^{\alpha}}} .
$$

This paper can be sorted as follows. In the next section, Characterizations based on the truncated moments, hazard function and conditional expectations are presented for the OGNH-G family. Section 3 provides some general mathematical properties of the family. Section 4 gives the estimation of the parameters of the family using the maximum likelihood method. In Section 5, some new special models of the generated family are considered. Some statistical properties, estimation and simulation study for odd generalized $\mathrm{NH}$ exponential model are derived in Section 6. The application of the odd generalized NH exponential distribution to a real data set is presented in Section 7. At the end, concluding remarks are outlined in Section 8.

\section{Characterizations of OGNH-G Distribution}

This section is devoted to the characterizations of the OGNH-G distribution in the following directions: (i) based on the ratio of two truncated moments and (ii) in terms of the hazard. Note that (i) can be employed also when the cdf does not have a closed form. We also like to point out that the characterization (i) is stable in the sense of weak convergence. We present our characterizations (i)-(ii) in two subsections.

\subsection{Characterizations based on two truncated moments}

This subsection is devoted to the characterizations of OGNH-G distribution based on the ratio of two truncated moments. Our first characterization employs a theorem due to Glänzel (1987), see Theorem 1 of Appendix A. The result, however, holds also when the interval $H$ is not closed, since the condition of the Theorem is on the interior of $H$. 
Proposition 2.1. Let $X: \Omega \rightarrow R$ be a continuous random variable and let $q_{1}(x)=\bar{G}(x ; \xi)^{\alpha+1}\left(1+\left(\frac{G(x ; \xi)}{\bar{G}(x ; \xi)}\right)^{\beta}\right)^{1-\alpha} \exp \left\{\left(1+\left(\frac{G(x ; \xi)}{\bar{G}(x ; \xi)}\right)^{\beta}\right)^{\alpha}\right\}$ and $q_{2}(x)=q_{1}(x) G(x ; \xi)^{\alpha}$ for $X \in \mathbb{R}$

The random variable $X$ has pdf (4) if and only if the function $\xi$ defined in Theorem 1 is of the form

$$
\xi(x)=\frac{1}{2}\left[1+G(x ; \xi)^{\alpha}\right], \quad X \in \mathbb{R} .
$$

Proof. Suppose the random variable $X$ has pdf (4), then

$$
(1-F(x)) E\left[q_{1}(x) / X \geq x\right]=C \beta\left[1-G(x ; \xi)^{\alpha}\right], \quad X \in \mathbb{R},
$$

and

$$
(1-F(x)) E\left[q_{2}(x) / X \geq x\right]=\frac{C \beta}{2}\left[1-G(x ; \xi)^{2 \alpha}\right], \quad X \in \mathbb{R}
$$

Further,

$$
\xi(x) q_{1}(x)-q_{2}(x)=\frac{q_{1}(x)}{2}\left[1-G(x ; \xi)^{\alpha}\right]>0, \quad \text { for } X \in \mathbb{R} .
$$

Conversely, if $\xi$ is of the above form, then

$$
S^{\prime}(x)=\frac{\xi^{\prime}(x) q_{1}(x)}{\xi(x) q_{1}(x)-q_{2}(x)}=\frac{\alpha g(x ; \xi) G(x ; \xi)^{\alpha-1}}{1-G(x ; \xi)^{\alpha}}, \quad X \in \mathbb{R}
$$

and consequently

$$
S(x)=-\log \left[1-G(x ; \xi)^{\alpha}\right], \quad X \in \mathbb{R}
$$

Now, according to Theorem $1, X$ has density (4).

Corollary 2.1. Let $X: \Omega \rightarrow R$ be a continuous random variable and let $q_{1}(x)$ be as in Proposition A.1. The random variable $X$ has pdf (4) if and only if there exist functions $q_{2}(x)$ and $\xi$ defined in Theorem 1 satisfying the following differential equation

$$
\frac{\xi^{\prime}(x) q_{1}(x)}{\xi(x) q_{1}(x)-q_{2}(x)}=\frac{\alpha g(x ; \xi) G(x ; \xi)^{\alpha-1}}{1-G(x ; \xi)^{\alpha}}, \quad X \in \mathbb{R}
$$

Corollary 2.2. The general solution of the differential equation in Corollary A.1 is

$$
\xi(x)=\left(1-G(x ; \phi)^{\alpha}\right)^{-1}\left[-\int \alpha g(x ; \xi) G(x ; \phi)^{\alpha-1}\left(q_{1}(x)\right)^{-1} q_{2}(x) d x+D\right],
$$

where $D$ is a constant. We like to point out that one set of functions satisfying the above differential equation is given in Proposition A.1 with $D=1 / 2$. Clearly, there are other triplets $\left(q_{1}, q_{2}, \xi\right)$ which satisfy conditions of Theorem 1.

\subsection{Characterization in terms of hazard function}

The hazard function, $h_{F}$, of a twice differentiable distribution function, $F$, satisfies the following first order differential equation

$$
\frac{f^{\prime}(x)}{f(x)}=\frac{h_{F}^{\prime}(x)}{h_{F}(x)}-h_{F}(x)
$$


It should be mentioned that for many univariate continuous distributions, the above equation is the only differential equation available in terms of the hazard function. In this subsection we present a non-trivial characterization of OGNH-G distribution in terms of the hazard function.

Proposition 2.2.1Let $X: \Omega \rightarrow R$ be a continuous random variable. The random variable $X$ has pdf (4) if and only if its hazard function $h_{F}$, satisfies the following differential equation

$$
h_{F}^{\prime}(x)-\frac{g^{\prime}(x ; \xi)}{g(x ; \xi)} h_{F}(x)=\alpha \beta g(x ; \xi) \frac{d}{d x}\left\{\frac{\left[G(x ; \xi)\left\{\bar{G}(x ; \xi)^{\beta}+G(x ; \xi)^{\beta}\right\}\right]^{\alpha-1}}{\bar{G}(x ; \xi)^{\alpha \beta+\alpha-\beta+1}}\right\}, \quad X \in \mathbb{R}
$$

Proof. If $X$ has pdf (4), then clearly the above differential equation holds. If the differential equation holds, then

$$
\frac{d}{d x}\left\{g(x ; \xi)^{-1} h_{F}(x)\right\}=\alpha \beta \frac{d}{d x}\left\{\frac{G(x ; \xi)^{\alpha-1}}{\bar{G}(x ; \xi)^{\alpha+1}}\left(1+\left(\frac{G(x ; \xi)}{\bar{G}(x ; \xi)}\right)^{\beta}\right)^{\alpha-1}\right\}
$$

from which we arrive at the hazard function corresponding to the pdf (4).

Remark 2.1. For $\beta=1$, we have a much simpler differential equation

$$
h_{F}^{\prime}(x ; \xi)-\frac{g^{\prime}(x ; \xi)}{g(x ; \xi)} h_{F}(x ; \xi)=\alpha(\alpha+1) g(x ; \xi)^{2} \bar{G}(x ; \xi)^{-\alpha-2}, X \in \mathbb{R}
$$

\section{Some Statistical Properties}

This section provides some statistical properties of OGNH-G family of distributions.

\subsection{Quantile function}

Let $X$ denotes a random variable has the pdf (4), the quantile function, say $Q(u)$ of $X$ is given by

$$
Q(u)=G^{-1}\left\{\frac{\left[1-[1-\ln [1-u]]^{\frac{1}{\alpha}}\right]^{\frac{1}{\beta}}}{1+\left[1-[1-\ln [1-u]]^{\frac{1}{\alpha}}\right]^{\frac{1}{\beta}}}\right\},
$$

where, $u$ is a uniform distribution on the interval $(0,1)$ and $\mathrm{G}^{-1}($.$) is the inverse function of \mathrm{G}($.$) .$

\subsection{Useful representation}

In this subsection, a useful expansion of the pdf and cdf for OGNH-G is provided.

Since the exponential series is

$$
e^{-\left(1+\left(\frac{G(x ; \zeta)}{1-G(x ; \zeta)}\right)^{\beta}\right)^{\alpha}}=\sum_{i=0}^{\infty} \frac{(-1)^{i}}{i !}\left(1+\left(\frac{G(x ; \zeta)}{1-G(x ; \zeta)}\right)^{\beta}\right)^{\alpha i}
$$

Inserting (6) in (4) then,

$$
f(x)=\alpha \beta C g(x ; \zeta) \frac{G(x ; \zeta)^{\beta-1}}{(1-G(x ; \zeta))^{\beta+1}} \sum_{i=0}^{\infty} \frac{(-1)^{i}}{i !}\left(1+\left(\frac{G(x ; \zeta)}{1-G(x ; \zeta)}\right)^{\beta}\right)^{\alpha(i+1)-1} .
$$

By using binomial theory

$$
(1+z)^{\beta}=\sum_{j=0}^{\infty} \quad\left(\begin{array}{c}
\beta \\
j
\end{array}\right) z^{j}
$$


for $|z|<1$, and $\beta$ is a positive real non integer. Then, by applying the binomial theorem (7)in the previous density function of OGNH-G family becomes

$$
f(x)=\alpha \beta C g(x ; \zeta) \sum_{i, j=0}^{\infty} \frac{(-1)^{i}}{i !}\left(\begin{array}{c}
\alpha(i+1)-1 \\
j
\end{array}\right) \frac{G(x ; \zeta)^{\beta(j+1)-1}}{(1-G(x ; \zeta))^{\beta(j+1)+1}} .
$$

Since, the binomial expansion

$$
(1-z)^{-\beta}=\sum_{k=0}^{\infty} \quad\left(\begin{array}{c}
\beta+k-1 \\
k
\end{array}\right) z^{k},
$$

By inserting (9) in (8) then, the pdf (8) can be written as follows

$$
f(x)=\sum_{k=0}^{\infty} \eta_{k} g(x ; \zeta) G(x ; \zeta)^{\beta(j+1)+k-1},
$$

where,

$$
\eta_{k}=\sum_{i, j=0}^{\infty} \frac{\alpha \beta C(-1)^{i}}{i !}\left(\begin{array}{c}
i \\
j
\end{array}\right)\left(\begin{array}{c}
\alpha(i+1)-1 \\
j
\end{array}\right)\left(\begin{array}{c}
\beta(j+1)+k \\
k
\end{array}\right) .
$$

Another formula can be extracted from pdf (10), which gives the following infinite linear combination

$$
f(x)=\sum_{k=0}^{\infty} W_{k} h_{\beta(j+1)+k}(x),
$$

where, $W_{k}=\frac{\eta_{k}}{(\beta(j+1)+k)}$, and $h_{a}(x)=a g(x ; \zeta) G(x ; \zeta)^{a-1}$, is the exponentiated-generated (exp-G) density with power parameter $a$. Further, an expansion for the $[F(x)]^{h}$ is derived, for $\mathrm{h}$ is integer, again, the binomial and exponential expansions is worked out.

where,

$$
[F(x)]^{h}=\sum_{z=0}^{\infty} S_{z} G(x ; \zeta)^{\beta u+z}
$$

$$
s_{z}=\sum_{i=0}^{h} \sum_{j, u=0}^{\infty} \frac{i^{j} C^{i}(-1)^{i+j}}{j !}\left(\begin{array}{c}
h \\
i
\end{array}\right)\left(\begin{array}{c}
\alpha j \\
u
\end{array}\right)\left(\begin{array}{c}
\beta u+z-1 \\
z
\end{array}\right) .
$$

\subsection{The probability weighted moments}

For a random variable $X$, the probability-weighted moments (PWMs), denoted by $\tau_{r, s}$, can be calculated through the following relation

$$
\tau_{r, s}=E\left[X^{r} F(x)^{s}\right]=\int_{-\infty}^{\infty} x^{r} f(x)(F(x))^{s} d x .
$$

The PWMs of OGNH-G is obtained by substituting (10) and (12) into (13), and replacing h with s, as follows

$$
\tau_{r, s}=\int_{-\infty}^{\infty} \sum_{k, z=0}^{\infty} s_{z} \eta_{k} x^{r} g(x ; \zeta)(G(x ; \zeta))^{\beta(j+u+1)+k+z-1} d x \text {. }
$$

Then,

$$
\tau_{r, s}=\sum_{k, Z=0}^{\infty} s_{z} \eta_{k} \tau_{r, \beta(j+u+1)+k+z-1},
$$

where, $\tau_{r, \beta(j+u+1)+k+z-1}=\int_{-\infty}^{\infty} x^{r} g(x ; \zeta)(G(x ; \zeta))^{\beta(j+u+1)+k+z-1} d x$.

\subsection{Moments}

Since the moments are necessary and important in any statistical analysis, especially in applications. Therefore, we derive the $r$ th moment for the OGNH-G family. If $X$ has the pdf (10), then $r$ thmoment is obtained as follows

$$
\mu_{r}^{\prime}=\int_{-\infty}^{\infty} x^{r} f(x) d x=\int_{-\infty}^{\infty} \sum_{k=0}^{\infty} \eta_{k} x^{r} g(x ; \zeta) G(x ; \zeta)^{\beta(j+1)+k-1} d x .
$$

Then,

$$
\mu_{r}^{\prime}=\sum_{k=0}^{\infty} \eta_{k} \tau_{r, \beta(j+1)+k-1} .
$$

For a random variable $X$, it is known that, the moment generating function is defined as 


$$
M_{X}(t)=\sum_{r=0}^{\infty} \quad \frac{t^{r}}{r !} \mu_{r}^{\prime}=\sum_{r, k=0}^{\infty} \frac{t^{r}}{r !} \eta_{k} \tau_{r, \beta(j+1)+k-1}
$$

\subsection{The mean deviation}

For random variable $X$ with $\operatorname{pdf} f(x), \operatorname{cdf} F(x)$, the mean deviation about the mean and mean deviation about the median, are defined by

$$
\delta_{1}=2 \mu F(\mu)-2 T(\mu) \text { and } \delta_{2}=\mu-2 T(M),
$$

where, $\mu=E(X), M=\operatorname{Median}(X)$, and $T(q)=\int_{-\infty}^{q} x f(x) d x$ which is the first incomplete moment.

\subsection{Order statistics}

Order statistics have been extensively applied in many fields of statistics, such as reliability and life testing. Let $X_{1}, X_{2}, \ldots, X_{n}$ be independent and identically distributed (i.i.d) random variables with their corresponding continuous distribution function $F(x)$. Let $X_{1: n}<X_{2: n}<\ldots<X_{n: n}$ the corresponding ordered random sample from a population of sizen. According to (David, 1981), the pdf of the sthorder statistic, is defined as

$$
f_{s: n}(x)=\frac{f(x)}{B(k, n-k+1)} \sum_{v=0}^{n-s} \quad(-1)^{v}\left(\begin{array}{c}
n-s \\
v
\end{array}\right) F(x)^{v+s-1},
$$

where, $B(.$, ) stands for beta function. The pdf of the sthorder statistic for OGNH-G family is derived by substituting (10) and (12) in (14), replacing h with $v+k-1$,

$$
f_{s: n}(x)=\frac{g(x ; \zeta)}{B(s, n-s+1)} \sum_{v=0}^{n-s} \quad \sum_{k, z=0}^{\infty} \eta_{i} p_{z, v} G(x ; \zeta)^{\beta(j+u+1)+k+z-1}
$$

where $p_{z, v}=(-1)^{v}\left(\begin{array}{c}n-s \\ v\end{array}\right) s_{z}, g($.$) and G($.$) are the pdf and cdf of the OGNH-G family, respectively. Further, the$ rthmoment of sthorder statistics for OGNH-G is defined family by:

$$
E\left(\begin{array}{ll}
X_{s: n} & r
\end{array}\right)=\int_{-\infty}^{\infty} x^{r} f_{s: n}(x) d x .
$$

By substituting (15) in (16), leads to

$$
E\left(X_{s: n}{ }^{r}\right)=\frac{1}{B(s, n-s+1)} \sum_{v=0}^{n-s} \sum_{k, z=0}^{\infty} \eta_{k} p_{z, v} \int_{-\infty}^{\infty} x^{r} g(x ; \zeta) G(x ; \zeta)^{\beta(j+u+1)+k+z-1} d x
$$

Then,

$$
E\left(X_{s: n}{ }^{r}\right)=\frac{1}{B(s, n-s+1)} \sum_{v=0}^{n-s} \sum_{k, z=0}^{\infty} \eta_{k} p_{z, v} \tau_{r, \beta(j+u+1)+k+z-1} .
$$

\subsection{Rényi entropy}

The entropy of a random variable $X$ is a measure of variation of uncertainty and has been used in many fields such as physics, engineering and economics. As mentioned by (Rényi 1961), the Rényi entropy is defined by

$$
I_{\delta}(X)=\frac{1}{1-\delta} \log \int_{-\infty}^{\infty} f(x)^{\delta} d x, \delta>0 \text { and } \delta \neq 1
$$

By applying the binomial and exponential theory in the pdf (4), then the pdf $f(x)^{\delta}$ can be expressed as follows

$$
f(x)^{\delta}=\sum_{k=0}^{\infty} t_{k} g(x ; \zeta)^{\delta} G(x ; \zeta)^{j+k}
$$

where

$$
t_{k}=\sum_{i, j=0}^{\infty} \frac{(\alpha \beta C)^{\delta}(-\delta)^{i}}{i !}\left(\begin{array}{c}
\alpha(i+\delta)-\delta \\
j
\end{array}\right)\left(\begin{array}{c}
\beta(j+\delta)+\delta+k-1 \\
k
\end{array}\right) .
$$

Therefore, the Rényi entropy of OGNH generated family of distributions is given by

$$
I_{\delta}(X)=\frac{1}{1-\delta} \log \sum_{k=0}^{\infty} t_{k} \int_{-\infty}^{\infty} g(x ; \zeta)^{\delta} G(x ; \zeta)^{\beta(j+\delta)+k-\delta} d x
$$




\section{Maximum Likelihood Method}

This section deals with the maximum likelihood estimators of the unknown parameters for the OGNH-G family of distributions on the basis of complete samples. Let $X_{1}, \ldots, X_{n}$ be the observed values from the ONH-G family with set of parameter $\Phi=(\alpha, \beta, \zeta)^{T}$. The log-likelihood function for parameter vector $\Phi=(\alpha, \beta, \zeta)^{T}$ is obtained as follows

$$
\begin{aligned}
& \ln L(\Phi)=n \ln \alpha+n \ln \beta+\sum_{i=1}^{n} \ln \left[g\left(x_{i} ; \zeta\right)\right]+(\beta-1) \sum_{i=1}^{n} \ln \left[G\left(x_{i} ; \zeta\right)\right]-(\beta+1) \sum_{i=1}^{n} \ln \left[1-G\left(x_{i} ; \zeta\right)\right] \\
& +(\alpha-1) \sum_{i=1}^{n} \ln \left[1+\left(\frac{G\left(x_{i} ; \zeta\right)}{1-G\left(x_{i} ; \zeta\right)}\right)^{\beta}\right]+\sum_{i=1}^{n}\left\{1-\left(1+\left(\frac{G\left(x_{i} ; \zeta\right)}{1-G\left(x_{i} ; \zeta\right)}\right)^{\beta}\right)^{\alpha}\right\} .
\end{aligned}
$$

The elements of the score function $U(\Phi)=\left(U_{\alpha}, U_{\beta}, U_{\zeta_{k}}\right)$ are given by

$$
\begin{aligned}
& U_{\alpha}=\frac{n}{\alpha}+\sum_{i=1}^{n} \ln \left[1+\left(\frac{G\left(x_{i} ; \zeta\right)}{1-G\left(x_{i} ; \zeta\right)}\right)^{\beta}\right]-\sum_{i=1}^{n}\left(1+\left(\frac{G\left(x_{i} ; \zeta\right)}{1-G\left(x_{i} ; \zeta\right)}\right)^{\beta}\right)^{\alpha} \ln \left(1+\left(\frac{G\left(x_{i} ; \zeta\right)}{1-G\left(x_{i} ; \zeta\right)}\right)^{\beta}\right) \\
& U_{\beta}=\frac{n}{\beta}+\sum_{i=1}^{n} \ln \left[G\left(x_{i} ; \zeta\right)\right]-\sum_{i=1}^{n} \ln \left[1-G\left(x_{i} ; \zeta\right)\right]+(\alpha-1) \sum_{i=1}^{n} \frac{\left(\frac{G\left(x_{i} ; \zeta\right)}{1-G\left(x_{i} ; \zeta\right)}\right)^{\beta} \ln \left(\frac{G\left(x_{i} ; \zeta\right)}{1-G\left(x_{i} ; \zeta\right)}\right)}{1+\left(\frac{G\left(x_{i} ; \zeta\right)}{1-G\left(x_{i} ; \zeta\right)}\right)^{\beta}} \\
& -\alpha \sum_{i=1}^{n}\left(1+\left(\frac{G\left(x_{i} ; \zeta\right)}{1-G\left(x_{i} ; \zeta\right)}\right)^{\beta}\right)^{\alpha-1}\left(\frac{G\left(x_{i} ; \zeta\right)}{1-G\left(x_{i} ; \zeta\right)}\right)^{\beta} \ln \left(\frac{G\left(x_{i} ; \zeta\right)}{1-G\left(x_{i} ; \zeta\right)}\right),
\end{aligned}
$$

and

$$
\begin{aligned}
& U_{\zeta_{k}}=\sum_{i=1}^{n} \frac{\partial g\left(x_{i} ; \zeta\right) / \partial \zeta_{k}}{g\left(x_{i} ; \zeta\right)}+(\beta-1) \sum_{i=1}^{n} \frac{\partial G\left(x_{i} ; \zeta\right) / \partial \zeta_{k}}{G\left(x_{i} ; \zeta\right)}+(\beta+1) \sum_{i=1}^{n} \frac{\partial G\left(x_{i} ; \zeta\right) / \partial \zeta_{k}}{\left(1-G\left(x_{i} ; \zeta\right)\right)} \\
& +\beta(\alpha-1) \sum_{i=1}^{n} \frac{G\left(x_{i} ; \zeta\right)^{\beta-1}}{\left(1-G\left(x_{i} ; \zeta\right)\right)^{\beta+1}} \partial G\left(x_{i} ; \zeta\right) / \partial \zeta_{k} \\
& 1+\left(\frac{G\left(x_{i} ; \zeta\right)}{1-G\left(x_{i} ; \zeta\right)}\right)^{\beta} \\
& -\alpha \beta \sum_{i=1}^{n}\left(1+\left(\frac{G\left(x_{i} ; \zeta\right)}{1-G\left(x_{i} ; \zeta\right)}\right)^{\beta}\right)^{\alpha-1} \frac{G\left(x_{i} ; \zeta\right)^{\beta-1}}{\left(1-G\left(x_{i} ; \zeta\right)\right)^{\beta+1}} \partial G\left(x_{i} ; \zeta\right) / \partial \zeta_{k} .
\end{aligned}
$$

Setting $U_{\alpha}, U_{\beta}$ and $U_{\zeta_{k}}$ equal to zero and solving these equations simultaneously yield the maximum likelihood estimate (MLE) $\widehat{\Phi}=(\hat{\alpha}, \hat{\beta}, \hat{\zeta})$ of $\Phi=(\alpha, \beta, \zeta)^{T}$. These equations cannot be solved analytically and statistical software can be used to solve them numerically using iterative methods.

\section{Special Models of the OGNH family}

In this section, we discuss four special models of the OGNH generated family, namely, OGNH-uniform (OGNHU), OGNH-Lomax (OGNHL), OGNH-Rayleigh (OGNHR) and OGNH-exponential (OGNHE) distributions, respectively. The plots of the pdf and hrf of each special model are also sketched for different parametric values.

\subsection{OGNH-Uniform Distribution}

Consider the pdf and cdf of the uniformly distributed random variable given by $g(x ; \theta)=\frac{1}{\theta}, \quad 0<x<\theta$, and $G(x ; \theta)=\frac{x}{\theta}$, respectively. Then the cdf of the OGNHU is given as

$$
F(x)=1-\exp \left\{1-\left(1+\left(\frac{x}{\theta-x}\right)^{\beta}\right)^{\alpha}\right\}, \quad x \alpha, \beta, \theta>0 .
$$

The pdf corresponding (3.1) takes the following form 


$$
f(x)=\frac{\alpha \beta \theta x^{\beta-1}}{(\theta-x)^{\beta+1}}\left(1+\left(\frac{x}{\theta-x}\right)^{\beta}\right)^{\alpha-1} \exp \left\{1-\left(1+\left(\frac{x}{\theta-x}\right)^{\beta}\right)^{\alpha}\right\}
$$

For different values of parameters, the plots of pdf and hrf for the OGNHU distribution are displayed in Figure 1 below:
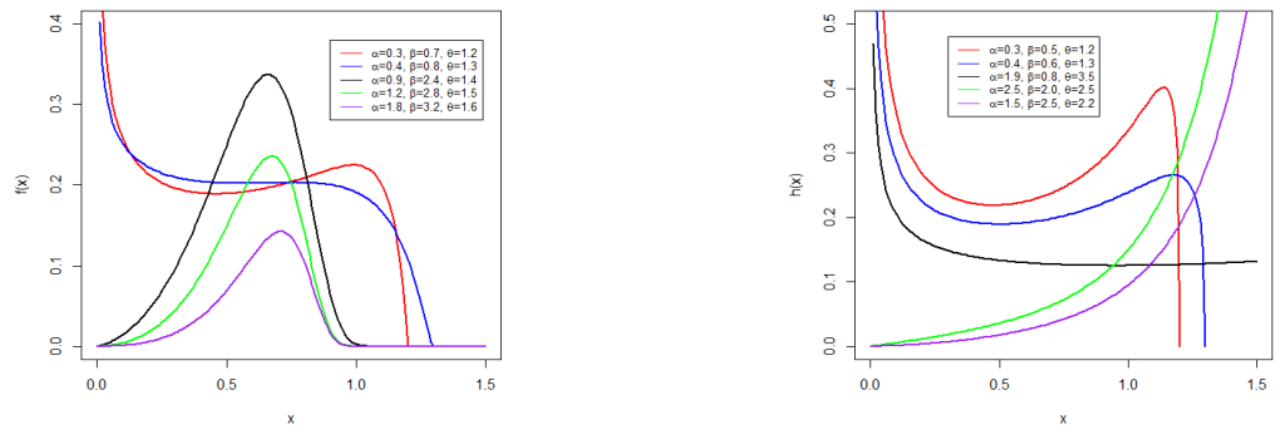

Figure 1: Pdf and hrf of OGNHU distribution for varying values of parameters.

\subsection{OGNH-Lomax Distribution}

Let the Lomax distribution be the parent distribution with pdf and cdf given by $g(x)=\theta \lambda(1+\lambda x)^{-\theta-1}, x, \theta, \lambda>0$, and $G(x)=1-(1+\lambda x)^{-\theta}$, respectively. Then, the cdf and pdf of the OGNHL random variable becomes

$$
F(x)=1-\exp \left\{1-\left(1+\left((1+\lambda x)^{\theta}-1\right)^{\beta}\right)^{\alpha}\right\}, \quad x, \alpha, \beta, \theta, \lambda>0,
$$

and

$f(x)=\frac{\alpha \beta \theta \lambda(1+\lambda x)^{\beta \theta-1}}{\left(1-(1+\lambda x)^{-\theta}\right)^{1-\beta}}\left(1+\left((1+\lambda x)^{\theta}-1\right)^{\beta}\right)^{\alpha-1} \exp \left\{1-\left(1+\left((1+\lambda x)^{\theta}-1\right)^{\beta}\right)^{\alpha}\right\}$.

For selected values of the model parameters, the graphs of pdf and hrf for the OGNHL distribution are illustrated in Figure 2 as:
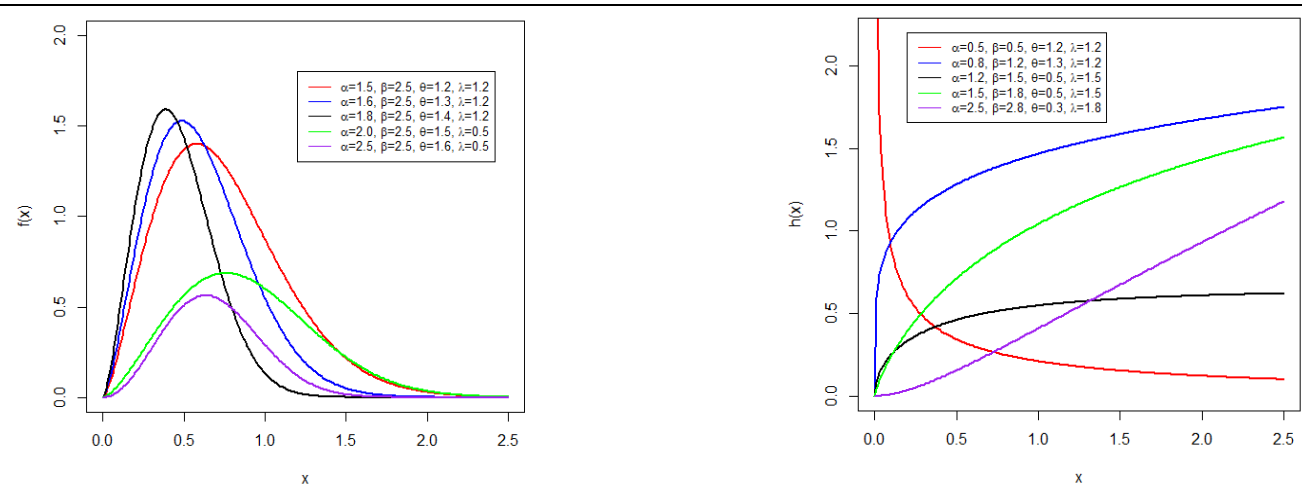

Figure 2: Pdf and hrf of OGNHL distribution for selected values of parmaeters. 


\subsection{OGNH- Rayleigh Distribution}

The pdf and cdf of the Rayleigh random variable has the following form $g(x)=2 \theta x e^{-\theta x^{2}}, x, \theta>0$, and $G(x)=$ $1-e^{-\theta x^{2}}$, respectively. Then, the cdf of OGNHR distribution is given by

$$
F(x)=1-\exp \left\{1-\left(1+\left(e^{\theta x^{2}}-1\right)^{\beta}\right)^{\alpha}\right\}, \quad x, \alpha, \beta, \theta>0
$$

with pdf

$$
f(x)=\frac{2 \alpha \beta \theta x e^{\theta \beta x^{2}}}{\left(1-e^{-\theta x^{2}}\right)^{1-\beta}}\left(1+\left(e^{\theta x^{2}}-1\right)^{\beta}\right)^{\alpha-1} \exp \left\{1-\left(1+\left(e^{\theta x^{2}}-1\right)^{\beta}\right)^{\alpha}\right\} .
$$

The graphical illustration of the pdf and hrf for the OGNHR are sketched below in Figure 3 as:

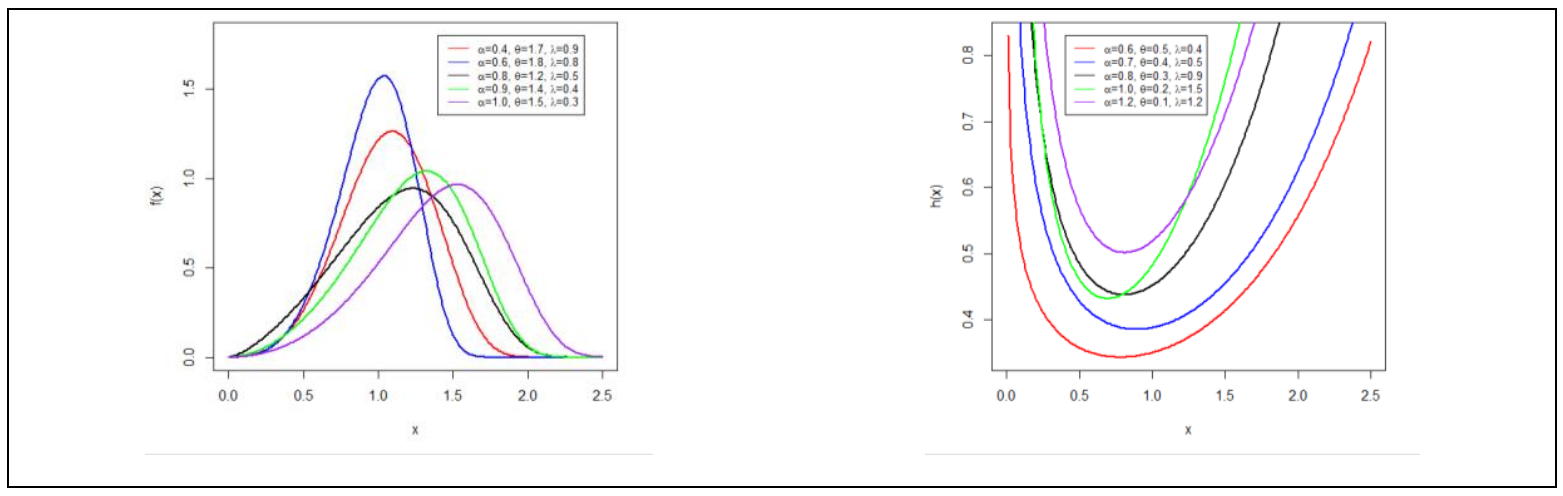

Figure 3: Pdf and hrf of OGNHR distribution for different values of parameters.

\subsection{OGNH-Exponential Distribution}

Considering the exponential distribution as a parent distribution with pdf and cdf given by $g(x)=\theta e^{-\theta x}, x, \theta>0$, and $G(x)=1-e^{-\theta x}$, respectively. Then, the cdf of OGNHE distribution is given by

$$
F(x)=1-\exp \left\{1-\left(1+\left(e^{\theta x}-1\right)^{\beta}\right)^{\alpha}\right\} .
$$

The density function corresponding to (3.7) becomes

$$
f(x)=\frac{\alpha \beta \theta e^{\theta \beta x}}{\left(1-e^{-\theta x}\right)^{1-\beta}}\left(1+\left(e^{\theta x}-1\right)^{\beta}\right)^{\alpha-1} \exp \left\{1-\left(1+\left(e^{\theta x}-1\right)^{\beta}\right)^{\alpha}\right\}, \quad x, \alpha, \beta, \theta>0,
$$

The graphical sketch of the pdf and hrf for the OGNHE are showed in Figure 4 as:

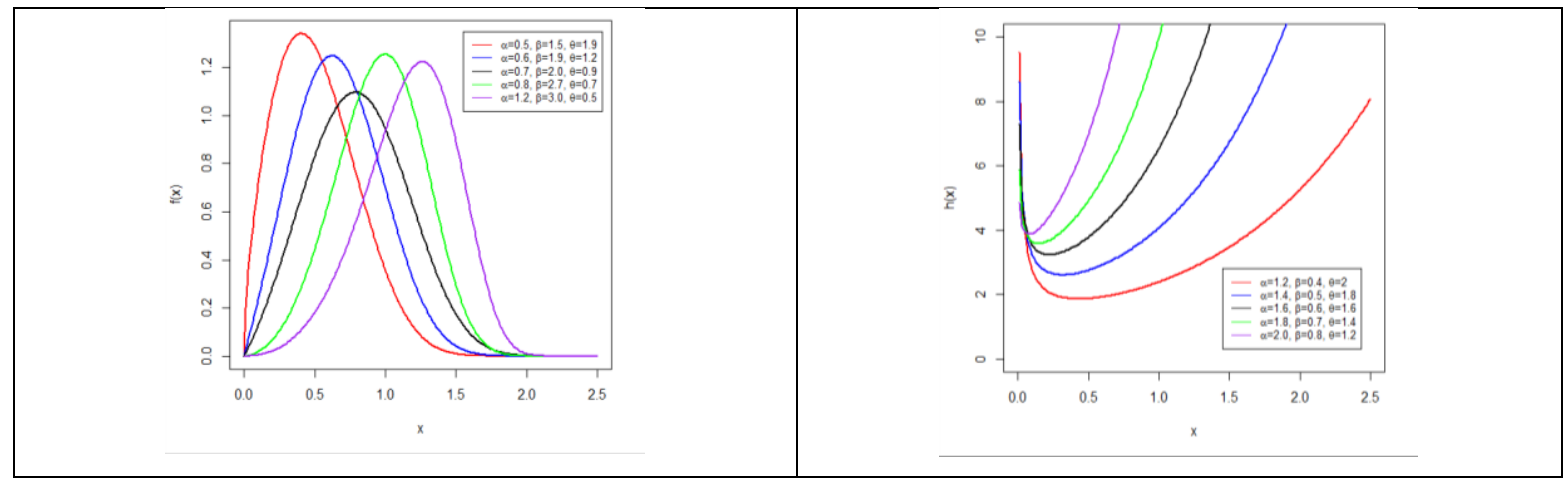

Figure 4: Pdf and hrf of OGNHE distribution for choice values of parameters. 


\section{Properties and Estimation of $O G N H E$ Distribution}

In this section, we derived fundamental properties, quantile function, moments, of $O G N H E$ distribution.

\subsection{Mathematical and statistical properties}

The quntile function of the $O G N H E$ distribution is given by

$$
x_{u}=\frac{1}{\theta} \ln \left[1+\left[[1-\ln [1-u]]^{\frac{1}{\alpha}}-1\right]^{\frac{1}{\beta}}\right]
$$

Median of the $O G N H E$ random variable is given by

\subsection{Mean deviations}

$$
M(X)=\frac{1}{\theta} \ln \left[1+\left[[1-\ln [0.5]]^{\frac{1}{\alpha}}-1\right]^{\frac{1}{\beta}}\right]
$$

For random variable $X$ with $\operatorname{pdf} f(x), \operatorname{cdf} F(x)$, the mean deviation about the mean and mean deviation about the median, are defined by

$$
\delta_{1}=2 \mu F(\mu)-2 T(\mu) \text { and } \delta_{2}=\mu-2 T(M),
$$

where, $\mu=E(X), M=\operatorname{Median}(X)$, and $T(q)=\sum_{\pi=0}^{\infty} \eta_{\pi} \frac{\gamma(2, q)}{((\pi+1) \theta)^{2}}$.

The probability-weighted moments of the $O G N H E$ distribution is given by

where

$$
\tau_{r, s}=\sum_{\pi, v=0}^{\infty} \eta_{\pi} s_{v} \frac{\Gamma(r+1)}{((\pi+v+1) \theta)^{r+1}}
$$

$$
\eta_{\pi}=\sum_{k=0}^{\infty} \theta(-1)^{\pi}\left(\begin{array}{c}
\beta(m+1)+k-1 \\
\pi
\end{array}\right) \theta \eta_{\pi}, s_{v}=\sum_{z=0}^{\infty}(-1)^{v}\left(\begin{array}{c}
u+z \\
v
\end{array}\right) s_{z} .
$$

The $r^{\text {th }}$ moment of the $O G N H E$ distribution is given by

$$
\mu_{r}^{\prime}=\sum_{\pi=0}^{\infty} \eta_{\pi} \frac{\Gamma(r+1)}{((\pi+1) \theta)^{r+1}} \text {. }
$$

The moment generating function of the OGNHE distribution is given by

$$
M_{X}(t)=\sum_{v, \pi=0}^{\infty} \frac{\eta_{\pi} t^{v}}{v !} \frac{\Gamma(r+1)}{((\pi+1) \theta)^{r+1}} .
$$

The pdf of the sthorder statistic is given by

$$
f_{s: n}(x)=\frac{1}{B(s, n-s+1)} \sum_{v=0}^{n-s} \sum_{\pi, v=0}^{\infty} \eta_{m} p_{z, v} e^{-\theta(\pi+v+1) x} .
$$

The rth moment of sthorder statistics of $O G N H E$ distribution is given by:

$$
E\left(X_{s: n}{ }^{r}\right)=\frac{1}{B(s, n-s+1)} \sum_{v=0}^{n-s} \sum_{\pi, v=0}^{\infty} \eta_{m} p_{z, v} \frac{\Gamma(r+1)}{((\pi+v+1) \theta)^{r+1}}
$$

\subsection{MLEs and their Performances}

The log-likelihood function for parameter vector $\Phi=(\alpha, \beta, \theta)^{T}$ is obtained as follows

$$
\begin{aligned}
& \ln L(\Phi)=n \ln \alpha+n \ln \beta+n \ln \theta+\theta \beta \sum_{i=1}^{n} x_{i}+(\beta-1) \sum_{i=1}^{n} \ln \left[1-e^{-\theta x_{i}}\right] \\
& +(\alpha-1) \sum_{i=1}^{n} \ln \left[1+\left(e^{\theta x_{i}}-1\right)^{\beta}\right]+\sum_{i=1}^{n}\left\{1-\left(1+\left(e^{\theta x_{i}}-1\right)^{\beta}\right)^{\alpha}\right\} .
\end{aligned}
$$

The components of score vector are: 


$$
\begin{aligned}
& U_{\alpha}=\frac{n}{\alpha}+\sum_{i=1}^{n} \ln \left[1+\left(e^{\theta x_{i}}-1\right)^{\beta}\right]-\sum_{i=1}^{n}\left(1+\left(e^{\theta x_{i}}-1\right)^{\beta}\right)^{\alpha} \ln \left(1+\left(e^{\theta x_{i}}-1\right)^{\beta}\right), \\
& U_{\beta}=\frac{n}{\beta}+\sum_{i=1}^{n} \ln \left[1-e^{-\theta x_{i}}\right]+\theta \sum_{i=1}^{n} x_{i}+(\alpha-1) \sum_{i=1}^{n} \frac{\left(e^{\theta x_{i}}-1\right)^{\beta} \ln \left(e^{\theta x_{i}}-1\right)}{1+\left(e^{\theta x_{i}}-1\right)^{\beta}} \\
& -\alpha \sum_{i=1}^{n}\left(1+\left(e^{\theta x_{i}}-1\right)^{\beta}\right)^{\alpha-1}\left(e^{\theta x_{i}}-1\right)^{\beta} \ln \left(e^{\theta x_{i}}-1\right),
\end{aligned}
$$

and

$$
\begin{aligned}
& U_{\theta}=\frac{n}{\theta}+\beta \sum_{i=1}^{n} x_{i}+(\beta-1) \sum_{i=1}^{n} \frac{x_{i} e^{-\theta x_{i}}}{1-e^{-\theta x_{i}}}+\beta(\alpha-1) \sum_{i=1}^{n} \frac{x_{i} e^{-\theta x_{i}}\left(e^{\theta x_{i}}-1\right)^{\beta-1}}{1+\left(e^{\theta x_{i}}-1\right)^{\beta}} \\
& -\alpha \beta \sum_{i=1}^{n} x_{i} e^{-\theta x_{i}}\left(1+\left(e^{\theta x_{i}}-1\right)^{\beta}\right)^{\alpha-1}\left(e^{\theta x_{i}}-1\right)^{\beta-1} .
\end{aligned}
$$

The above equations cannot be solved analytically; rather analytical software is required to solve them numerically. Further, a numerical investigation is carried out to evaluate the performance of ML estimators for ONHE model. Performance of estimators is evaluated through their biases, and mean square errors (MSEs) for different sample sizes. A numerical study is performed using Mathematica (9) software. Different sample sizes are considered through the experiments at size $\mathrm{n}=50,100,150$ and 200. In addition, the different values of parameters $(\alpha, \beta, \theta)$ are considered. The experiment is repeated 10000 times. In each experiment, the estimates of the parameters are obtained by ML method of estimation. The MSEs and biases for the different estimates are reported from these experiments in Table 1.

Table 1: The MSEs and Biases of ML estimates for the parameters of the ONHE distribution.

\begin{tabular}{|c|c|c|c|c|c|c|c|}
\hline & & \multicolumn{3}{|c|}{$(0.5,0.5,0.5)$} & \multicolumn{3}{c|}{$(1.5,0.5,0.5)$} \\
\hline$n$ & Par & MLEs & Bias & MSE & MLEs & Bias & MSE \\
\hline \multirow{3}{*}{50} & $\alpha$ & 0.5274 & 0.0274 & 0.0177 & 1.5854 & 0.0854 & 0.1536 \\
\cline { 2 - 8 } & $\beta$ & 0.5261 & 0.0261 & 0.0167 & 0.5177 & 0.0177 & 0.0096 \\
\cline { 2 - 8 } & $\theta$ & 0.5199 & 0.0199 & 0.0115 & 0.5080 & 0.0080 & 0.0039 \\
\hline \multirow{3}{*}{100} & $\alpha$ & 0.5142 & 0.0142 & 0.0078 & 1.5456 & 0.0456 & 0.0684 \\
\cline { 2 - 8 } & $\beta$ & 0.5132 & 0.0132 & 0.0075 & 0.5077 & 0.0077 & 0.0044 \\
\cline { 2 - 8 } 150 & $\theta$ & 0.5106 & 0.0106 & 0.0056 & 0.5047 & 0.0047 & 0.0020 \\
\cline { 2 - 8 } & $\alpha$ & 0.5098 & 0.0098 & 0.0051 & 1.5257 & 0.0257 & 0.0451 \\
\cline { 2 - 8 } & $\beta$ & 0.5091 & 0.0091 & 0.0049 & 0.5042 & 0.0042 & 0.0028 \\
\hline \multirow{3}{*}{200} & $\alpha$ & 0.5074 & 0.0074 & 0.0037 & 0.5023 & 0.0023 & 0.0014 \\
\cline { 2 - 8 } & $\beta$ & 0.5074 & 0.0074 & 0.0036 & 1.5174 & 0.0174 & 0.0329 \\
\cline { 2 - 8 } & $\theta$ & 0.5057 & 0.0075 & 0.0035 & 0.5042 & 0.0042 & 0.0021 \\
\hline
\end{tabular}




\section{Continued of Table 1}

\begin{tabular}{|c|c|c|c|c|c|c|c|}
\hline & & \multicolumn{3}{|c|}{$(2,0.5,0.5)$} & \multicolumn{3}{c|}{$(0.5,1.5,0.5)$} \\
\hline$n$ & Par & MLEs & Bias & MSE & MLEs & Bias & MSE \\
\hline \multirow{3}{*}{50} & $\alpha$ & 2.1127 & 0.1127 & 0.2779 & 0.5301 & 0.0301 & 0.0176 \\
\cline { 2 - 8 } & $\beta$ & 0.5203 & 0.0203 & 0.0120 & 1.5894 & 0.0894 & 0.1548 \\
\cline { 2 - 8 } & $\theta$ & 0.5064 & 0.0064 & 0.0033 & 0.5492 & 0.0492 & 0.0369 \\
\hline \multirow{3}{*}{100} & $\alpha$ & 2.0533 & 0.0533 & 0.1203 & 0.5136 & 0.0136 & 0.0079 \\
\cline { 2 - 8 } & $\beta$ & 0.5098 & 0.0098 & 0.0051 & 1.5406 & 0.0406 & 0.0698 \\
\cline { 2 - 8 } 150 & $\theta$ & 0.5031 & 0.0031 & 0.0016 & 0.5215 & 0.0215 & 0.0137 \\
\hline \multirow{3}{*}{200} & $\alpha$ & 2.0354 & 0.0354 & 0.0813 & 0.5089 & 0.0089 & 0.0047 \\
\cline { 2 - 8 } & $\beta$ & 0.5052 & 0.0052 & 0.0032 & 1.5264 & 0.0264 & 0.0416 \\
\cline { 2 - 8 } & $\alpha$ & 0.5019 & 0.0019 & 0.0012 & 0.5137 & 0.0137 & 0.0077 \\
\cline { 2 - 8 } & $\beta$ & 0.0269 & 0.0269 & 0.0571 & 0.5066 & 0.0066 & 0.0036 \\
\hline
\end{tabular}

Continued of Table 1

\begin{tabular}{|c|c|c|c|c|c|c|c|}
\hline & & \multicolumn{3}{|c|}{$(0.5,2,0.5)$} & \multicolumn{3}{c|}{$(1.5,1.5,0.5)$} \\
\hline$n$ & Par & MLEs & Bias & MSE & MLEs & Bias & MSE \\
\hline \multirow{3}{*}{50} & $\alpha$ & 0.5255 & 0.0255 & 0.0166 & 1.5857 & 0.0857 & 0.1570 \\
\cline { 2 - 8 } & $\beta$ & 2.1000 & 0.0999 & 0.2580 & 1.5514 & 0.0514 & 0.0849 \\
\cline { 2 - 8 } & $\theta$ & 0.5737 & 0.0737 & 0.1805 & 0.5770 & 0.0770 & 0.1049 \\
\hline \multirow{3}{*}{100} & $\alpha$ & 0.5132 & 0.0132 & 0.0076 & 1.5447 & 0.0447 & 0.0691 \\
\cline { 2 - 8 } & $\beta$ & 2.0522 & 0.0522 & 0.1186 & 1.5235 & 0.0234 & 0.0396 \\
\cline { 2 - 8 } 150 & $\theta$ & 0.5330 & 0.0330 & 0.0248 & 0.5346 & 0.0346 & 0.0229 \\
\hline \multirow{3}{*}{200} & $\alpha$ & 0.5094 & 0.0094 & 0.0048 & 1.5279 & 0.0279 & 0.0438 \\
\cline { 2 - 8 } & $\beta$ & 2.0363 & 0.0363 & 0.0750 & 1.5163 & 0.0163 & 0.0260 \\
\cline { 2 - 8 } & $\alpha$ & 0.5220 & 0.0220 & 0.0134 & 0.5212 & 0.0212 & 0.0128 \\
\cline { 2 - 8 } & $\beta$ & 0.5075 & 0.0075 & 0.0037 & 1.5158 & 0.0158 & 0.0312 \\
\hline
\end{tabular}

\section{Real Life Applications}

In this section, we have provided two applications using real data sets to illustrate efficiency of the new proposal. The first data set taken from Murthy et al. (2004) representing the failure times, while the second data set taken from the website: http://www.ceramics.nist.gov/srd/summary/ftmain.htm representing the fracture toughness of Alumina (Al2O3). For the interest of readers, we have provided the data in Table 2, and summarized in Table 3, as follows 
Table 2: Failure times and fracture toughness data sets.

\begin{tabular}{ll}
\hline & $0.036,0.058,0.061,0.074,0.078,0.086,0.102,0.103,0.114,0.116,0.148$, \\
& $0.183,0.192,0.254,0.262,0.379,0.381,0.538,0.570,0.574,0.590,0.618$, \\
Data 1 & $0.645,0.961,1.228,1.600,2.006,2.054,2.804,3.058,3.076,3.147,3.625$, \\
Failure & $3.704,3.931,4.073,4.393,4.534,4.893,6.274,6.816,7.896,7.904,8.022$, \\
times data & $9.337,10.940,11.020,13.880,14.730,15.080$ \\
\hline & $5.5,5,4.9,6.4,5.1,5.2,5.2,5,4.7,4,4.5,4.2,4.1,4.56,5.01,4.7,3.13,3.12$, \\
& $2.68,2.77,2.7,2.36,4.38,5.73,4.35,6.81,1.91,2.66,2.61,1.68,2.04,2.08$, \\
Data 2 & $2.13,3.8,3.73,3.71,3.28,3.9,4,3.8,4.1,3.9,4.05,4,3.95,4,4.5,4.5,4.2,4.55$, \\
Alumina & $4.65,4.1,4.25,4.3,4.5,4.7,5.15,4.3,4.5,4.9,5,5.35,5.15,5.25,5.8,5.85,5.9$, \\
(Al2O3) & $5.75,6.25,6.05,5.9,3.6,4.1,4.5,5.3,4.85,5.3,5.45,5.1,5.3,5.2,5.3,5.25$, \\
& $4.75,4.5,4.2,4,4.15,4.25,4.3,3.75,3.95,3.51,4.13,5.4,5,2.1,4.6,3.2,2.5$, \\
& $4.1,3.5,3.2,3.3,4.6,4.3,4.3,4.5,5.5,4.6,4.9,4.3,3,3.4,3.7,4.4,4.9,4.9,5$ \\
\hline
\end{tabular}

Table 3: Descriptive measures of the data given in table 1.

\begin{tabular}{ccccccc}
\hline Summary Statistics & Min. & $1^{\text {st }}$ Quartile & Median & Mean & $3^{\text {rd }}$ Quartile & Max. \\
\hline Data 1 & 0.0360 & 0.2075 & 1.4140 & 3.3430 & 4.4990 & 15.0800 \\
Data 2 & 1.680 & 3.850 & 4.380 & 4.325 & 5.000 & 6.810 \\
\hline
\end{tabular}

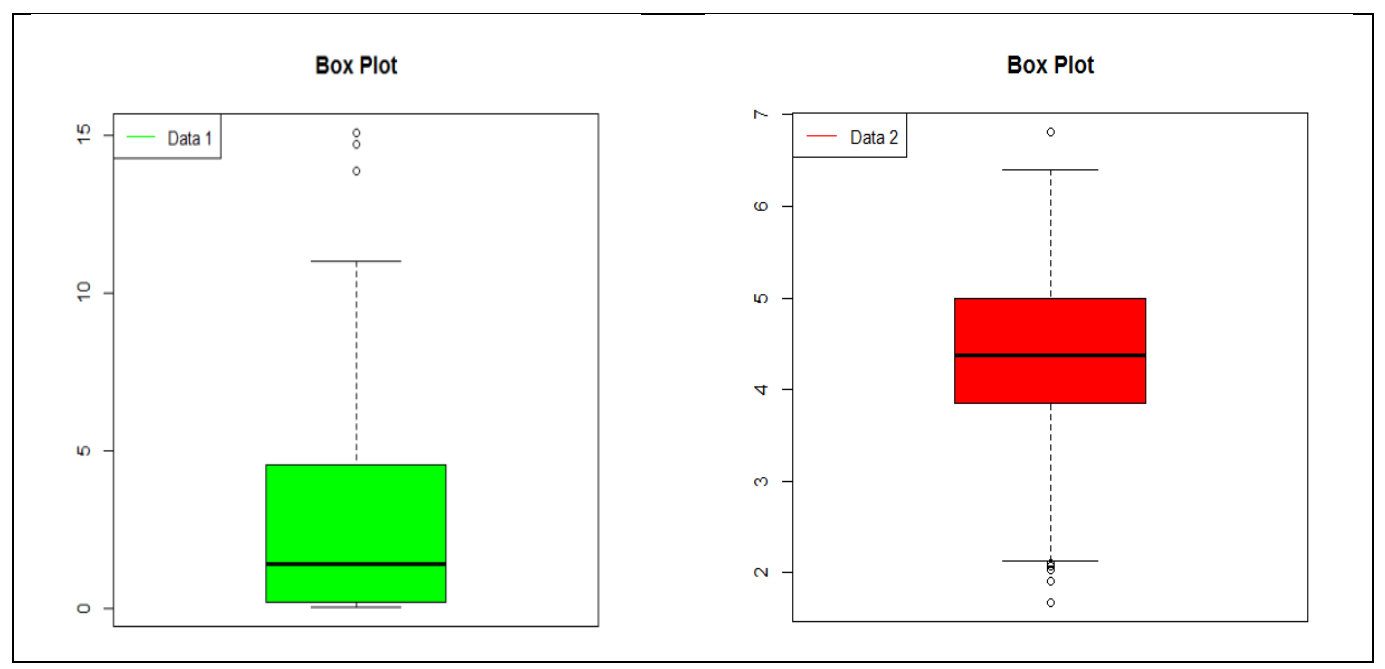

Figure 5: Box plots of the data given in table 1.

We compare the efficiency of the new proposal to some well-known lifetime distributions, namely; generalized linear exponential (GLE), exponentiated generalized linear exponential (Ex-GLE), exponential Lomax (EL), alpha power exponential (APE) and alpha power transformed Weibull (APTW) distributions. The cdfs of the other fitted models are

Generalized linear exponential of Mahmoud and Alam (2010)

$$
G(x)=1-e^{-\left(\alpha x^{2}+\beta x\right)^{\theta}} \quad x, \alpha, \beta, \theta>0 .
$$

Exponentiated generalized linear exponential by Sarhanet al. (2013)

$$
G(x)=\left(1-e^{-\left(\alpha x^{2}+\beta x\right)^{\theta}}\right)^{\lambda} \quad x, \alpha, \beta, \theta, \lambda>0 .
$$


Exponential Lomax studied proposed by El-Bassiouny et al. (2015)G(x)=1- $e^{-\theta\left(\frac{\beta}{x+\beta}\right)^{-\alpha}}, \quad x \geq$ $-\beta, \alpha, \beta, \theta>0$.

Alpha power exponential studied by Mahdavi and Kundu (2017)

$$
G(x)=\frac{\alpha^{\left(1-e^{-\beta x}\right)}-1}{\alpha-1}, \quad x, \alpha, \beta>0, \alpha \neq 1 .
$$

Alpha power transformed Weibull introduced by Dey et al. (2017)

$$
G(x)=\frac{\alpha^{\left(1-e^{-\beta x^{\theta}}\right)}-1}{\alpha-1}, \quad \quad x, \alpha, \beta, \theta>0, \alpha \neq 1 .
$$

To show the goodness of fit of the fitted models, we consider the analytical measures including Kolmogorov-Smirnov (KS) statistic, Anderson Darling (AD) statistic, Cramer-Von-Misses (CM) statistic, log-likelihood (LL), Akaike Information Criterion (AIC), Bayesian information criterion (BIC), corrected Akaike information criterion (CAIC) and Hannan-Quinn information criterion (HQIC). The analytical measure are given in Table 4, 5, 6 and 7 shows that the suggested method provides best fit than the other fitted distributions.

\begin{tabular}{|c|c|c|c|c|}
\hline Dist. & $\hat{\alpha}$ & $\hat{\beta}$ & $\widehat{\theta}$ & $\hat{\lambda}$ \\
\hline OGNHE & $\begin{array}{c}0.481 \\
(0.4013)\end{array}$ & $\begin{array}{c}0.497 \\
(0.0632)\end{array}$ & $\begin{array}{c}0.148 \\
(0.0859)\end{array}$ & \\
\hline GLE & $\begin{array}{c}0.015 \\
(0.018)\end{array}$ & $\begin{array}{c}0.535 \\
(0.1170)\end{array}$ & $\begin{array}{c}0.589 \\
(0.0915)\end{array}$ & \\
\hline APTW & $\begin{array}{c}0.798 \\
(1.164)\end{array}$ & $\begin{array}{c}0.500 \\
(0.2626)\end{array}$ & $\begin{array}{c}0.669 \\
(0.1146)\end{array}$ & \\
\hline Ex-GLE & $\begin{array}{c}6.578 \\
(10.829)\end{array}$ & $\begin{array}{r}0.688 \\
(1.462)\end{array}$ & $\begin{array}{c}0.274 \\
(0.0758)\end{array}$ & $\begin{array}{c}4.287 \\
(2.5937)\end{array}$ \\
\hline EL & $\begin{array}{c}0.609 \\
(0.0647)\end{array}$ & $\begin{array}{c}0.0037 \\
(0.00146)\end{array}$ & $\begin{array}{c}0.190 \\
(0.0095)\end{array}$ & \\
\hline APTE & $\begin{array}{c}0.531 \\
(0.1336)\end{array}$ & $\begin{array}{c}0.589 \\
(0.0629)\end{array}$ & & \\
\hline
\end{tabular}

Table 4: Maximum likelihood estimates and their respective standard errors in the parenthesis for data 1.

Table 5: Analytical results of the proposed and other competing models for data 1.

\begin{tabular}{ccccccccc}
\hline Dist. & KS & AD & CM & LL & AIC & BIC & CIAC & HQIC \\
\hline OGNHE & 0.139 & 0.871 & 0.129 & 101.52 & 209.05 & 214.79 & 209.57 & 211.24 \\
GLE & 0.122 & 0.885 & 0.131 & 101.77 & 209.54 & 215.28 & 210.07 & 211.73 \\
APTW & 0.140 & 0.892 & 0.133 & 102.12 & 210.64 & 216.38 & 211.16 & 212.83 \\
Ex-GLE & 0.115 & 0.896 & 0.139 & 102.28 & 210.57 & 217.22 & 211.46 & 213.48 \\
EL & 0.142 & 0.975 & 0.155 & 102.96 & 211.93 & 217.66 & 212.45 & 214.11 \\
APTE & 0.220 & 1.027 & 0.165 & 107.09 & 218.19 & 222.01 & 218.45 & 219.65 \\
\hline
\end{tabular}




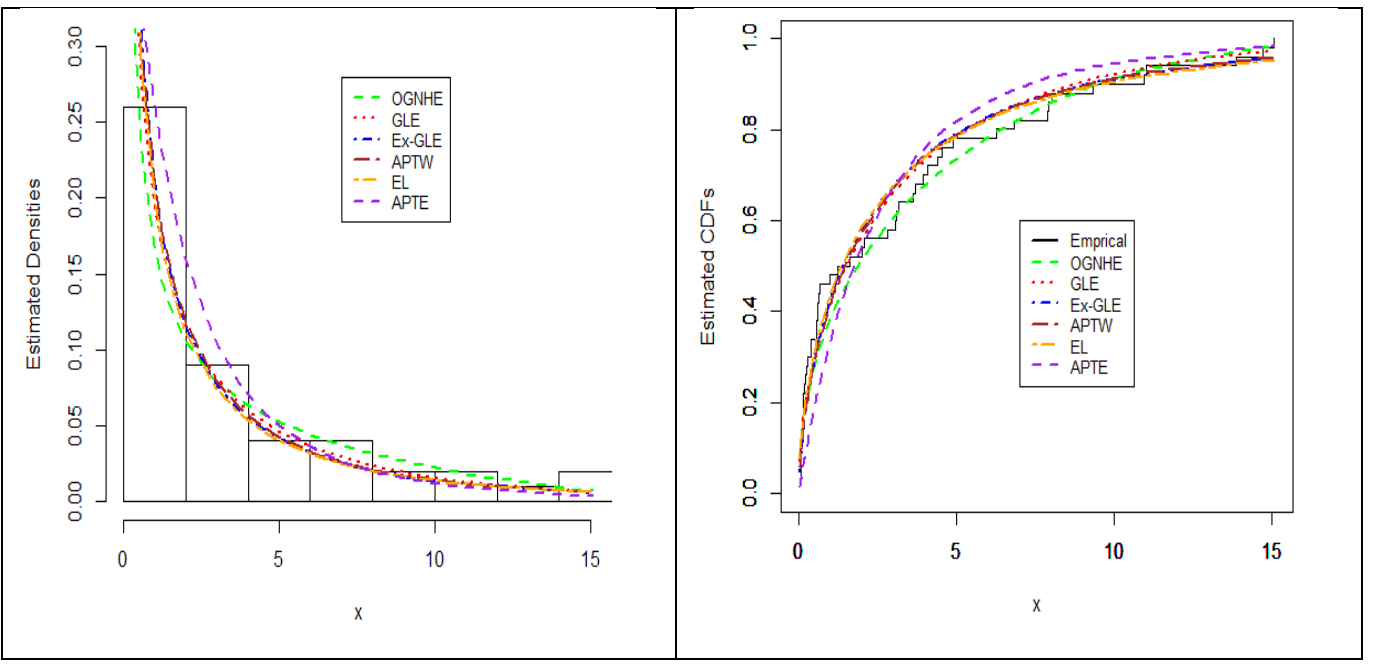

Figure 6: Estimated pdfs and cdfs of the fitted models corresponding to data 1.

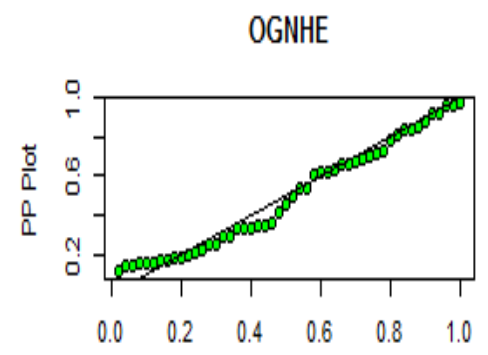

$\mathrm{X}$

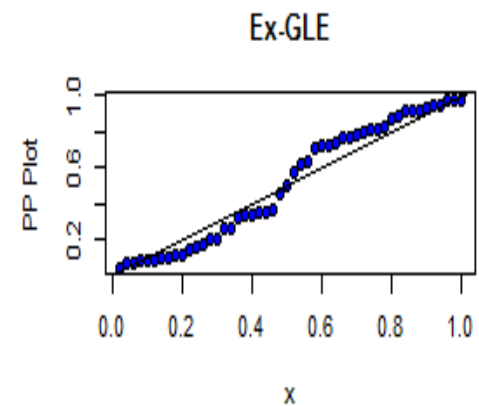

EL

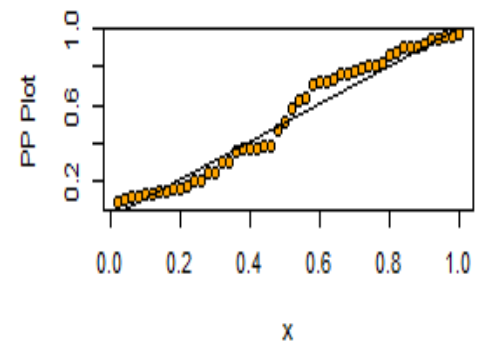

GLE

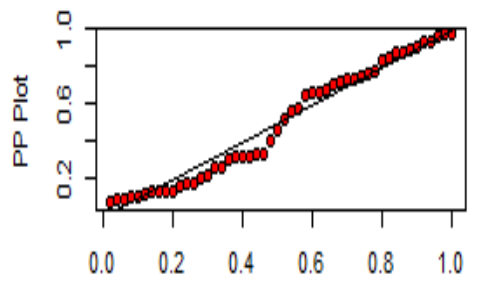

$\mathrm{x}$

APTW

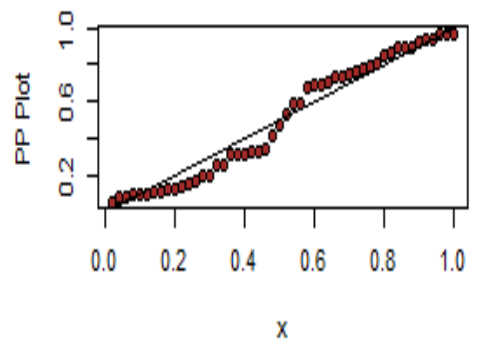

APTE

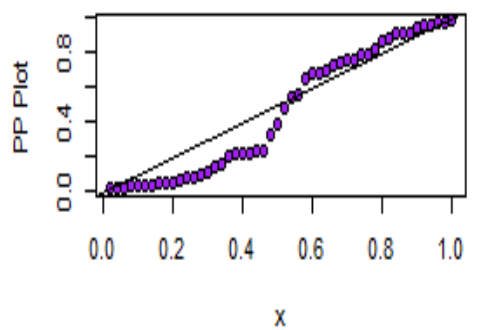

Figure 7: PP plots of the fitted models corresponding to data 1. 
Table 6: Maximum likelihood estimates and their respective standard errors in the parenthesis for data 2.

\begin{tabular}{|c|c|c|c|c|}
\hline Dist. & $\hat{\alpha}$ & $\hat{\beta}$ & $\hat{\theta}$ & $\hat{\lambda}$ \\
\hline OGNHE & $\begin{array}{c}0.974 \\
(0.3625)\end{array}$ & $\begin{array}{c}0.641 \\
(0.4203)\end{array}$ & $\begin{array}{c}0.1462 \\
(0.0159)\end{array}$ & \\
\hline GLE & $\begin{array}{r}0.0420 \\
(0.5398)\end{array}$ & $\begin{array}{c}0.0542 \\
(0.0118)\end{array}$ & $\begin{array}{c}2.0655 \\
(0.3070)\end{array}$ & \\
\hline APTW & $\begin{array}{r}2.3290 \\
(0.7308)\end{array}$ & $\begin{array}{c}0.0329 \\
(0.0789)\end{array}$ & $\begin{array}{c}3.9312 \\
(0.1318)\end{array}$ & \\
\hline Ex-GLE & $\begin{array}{r}0.0064 \\
(0.1245)\end{array}$ & $\begin{array}{c}3.0452 \\
(0.5231)\end{array}$ & $\begin{array}{l}2.3923 \\
(0.5983)\end{array}$ & $\begin{array}{c}1.0535 \\
(0.4834)\end{array}$ \\
\hline EL & $\begin{array}{r}14.7402 \\
(3.1329)\end{array}$ & $\begin{array}{c}9.8520 \\
(2.2892)\end{array}$ & $\begin{array}{c}0.0039 \\
(0.0059)\end{array}$ & \\
\hline APTE & $\begin{array}{c}21.442 \\
(6.488)\end{array}$ & $\begin{array}{c}0.4295 \\
(3.0274)\end{array}$ & & \\
\hline
\end{tabular}

Table 7: Analytical results of the proposed and other competing models for data 2.

\begin{tabular}{ccccccccc}
\hline Dist. & KS & AD & CM & LL & AIC & BIC & CIAC & HQIC \\
\hline OGNHE & 0.063 & 0.473 & 0.078 & 168.64 & 343.28 & 351.62 & 343.49 & 346.67 \\
GLE & 0.092 & 0.516 & 0.802 & 168.70 & 345.17 & 353.51 & 345.38 & 348.56 \\
APTW & 0.144 & 0.573 & 0.087 & 170.60 & 347.21 & 355.55 & 347.42 & 350.60 \\
Ex-GLE & 0.087 & 0.565 & 0.084 & 168.84 & 345.68 & 354.79 & 346.03 & 350.19 \\
EL & 0.093 & 0.591 & 0.149 & 170.82 & 347.65 & 355.99 & 347.86 & 351.03 \\
APTE & 0.310 & 2.041 & 0.334 & 245.59 & 495.18 & 500.74 & 495.28 & 497.44 \\
\hline
\end{tabular}

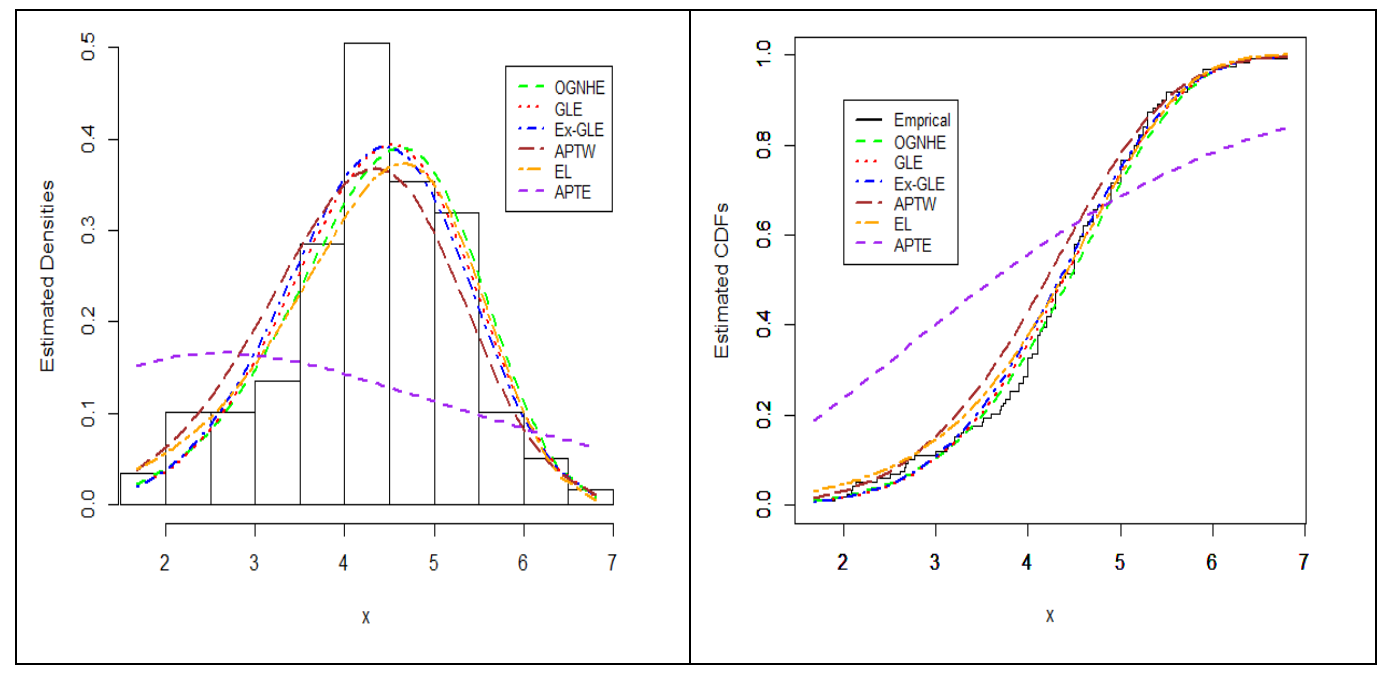

Figure 8: Estimated pdfs and cdfs of the fitted models resultant to data 2. 
OGNHE

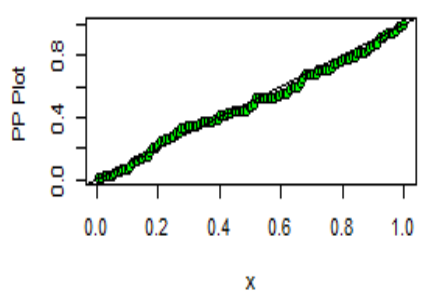

Ex-GLE

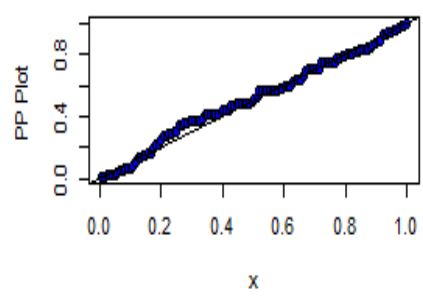

EL

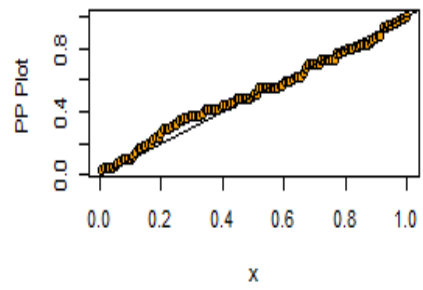

GLE

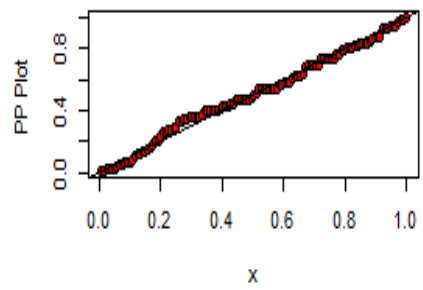

APTW

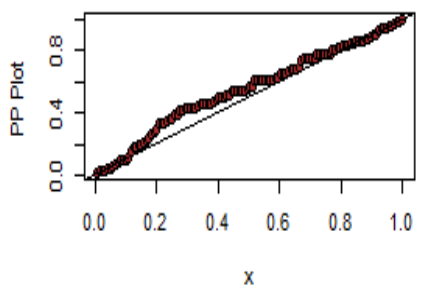

APTE

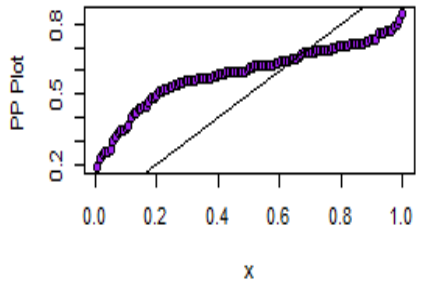

Figure 9: PP plots of the fitted models corresponding to data 2.

\section{Conclusion}

In this paper, we introduce the odd generalized NH-G family. Some of its properties are derived and some members of the family are defined. A member of the odd generalized $\mathrm{NH}-\mathrm{G}$ family, namely, the odd generalized $\mathrm{NH}$ exponential distribution is defined and studied. Various properties of the odd generalized NH exponential distribution including, probability weighted moments, moments, Rényi entropy and order statistics are derived. Estimation and simulation issues for the $O G N H E$ model are performed to study the behavior of the estimated parameters. The $O G N H E$ is applied to fit a real data set. This application shows that the $O G N H E$ can be preferred over some other well-known distributions.

\section{Appendix A}

Theorem 1 . Let $(\Omega, F, P)$ be a given probability space and let $H=[a, b]$ be an interval for some $d<b(a=-\infty, b=$ $\infty$ might as well be allowed).

Let $X: \Omega \rightarrow H$ be a continuous random variable with the distribution function $F$ and let $q_{1}$ and $q_{2}$ be two real functions defined on $H$ such that

$$
E\left[q_{2}(x) / X \geq x\right]=E\left[q_{1}(x) / X \geq x\right] \xi(x) \quad X \in H,
$$


is defined with some real function $\eta$. Assume that $q_{1}, q_{2}, C^{1}(H), \xi \in C^{2}(H)$ and $F$ is twice continuously differentiable and strictly monotone function on the set $H$. Finally, assume that the equation $\xi q_{1}=q_{2}$ has no real solution in the interior of $H$. Then $F$ is uniquely determined by the functions $q_{1}, q_{2}$ and $\xi$, particularly.

$$
F(x)=\int_{a}^{x} C\left|\frac{\xi^{\prime}(u)}{\xi(u) q_{1}(u)-q_{2}(u)}\right| \exp (-S(u)) d u,
$$

Where the function $S$ is a solution of the differential equation $S^{\prime}=\frac{\xi / q_{1}}{\xi q_{1-} q_{2}}$ and $C$ is the normalization constant, such that $\int_{H} d F=1$.

We like to mention that this kind of characterization based on the ratio of truncated moments is stable in the sense of weak convergence (see, Glänzel [2]), in particular, let us assume that there is a sequence $\left\{X_{n}\right\}$ of random variables with distribution functions $\left\{F_{n}\right\}$ such that the functions $q_{1 n}, q_{2 n}$ and $\xi_{n},(n \in \mathbb{N})$ satisfy the conditions of Theorem 1 and let $q_{1 n} \rightarrow q_{1}, q_{2 n} \rightarrow q_{2}$ for some continuously differentiable real functions $q_{1}$ and $q_{2}$. Let, finally, $X$ be a random variable with distribution $F$. Under the condition that $q_{1 n} X_{n}$ and $q_{2 n} X_{n}$ are uniformly integrable and the family $\left\{F_{n}\right\}$ is relatively compact, the sequence $X_{n}$ converges to $X$ in distribution if and only if $\xi_{n}$ converges to $\xi$, where

$$
\xi(x)=\frac{E\left[q_{2}(x) / X \geq x\right]}{E\left[q_{1}(x) / X \geq x\right]}
$$

This stability theorem makes sure that the convergence of distribution functions is reflected by corresponding convergence of the functions $q_{1}, q_{2}$ and $\xi$, respectively. It guarantees, for instance, the 'convergence' of characterization of the Wald distribution to that of the Lévy-Smirnov distribution if $\alpha \rightarrow \infty$.

A further consequence of the stability property of Theorem 1 is the application of this theorem to special tasks in statistical practice such as the estimation of the parameters of discrete distributions. For such purpose, the functions $q_{1}, q_{2}$ and, specially, $\xi$ should be as simple as possible. Since the function triplet is not uniquely determined it is often possible to choose $\xi$ as a linear function. Therefore, it is worth analyzing some special cases which helps to find new characterizations reflecting the relationship between individual continuous univariate distributions and appropriate in other areas of statistics.

\section{References}

1. Alizadeh, M., Cordeiro, G. M., Nascimento, A. D. C, Lima, M. C. S., and Ortega, E. M. M. (2017). Odd-Burr generalized family of distributions with some applications. Journal of Statistical Computation \& Simulation, 87(2), 367-389.

2. Alzaatreh, A., Lee, C. and Famoye, F. (2013). A new method for generating families of continuous distributions. Metron, 71, 63-79.

3. Bourguignon, M., Silva, R.B. and Cordeiro, G. M. (2014). The Weibull- G family of probability distributions. Journal of Data Science, 12, 53-68.

4. Cordeiro, G. M. and de Castro, M. (2011). A new family of generalized distribution. Journal of Statistical Computations and Simulation, 81, 883-898.

5. Cordeiro, G. M., Alizadeh, M. and Ortega, E. M. M. (2014). The exponentiated half-logistic family of distributions: Properties and applications. Journal of Probability and Statistics,Article ID 864396, 21 pages. http://dx.doi.org/10.1155/2014/864396.

6. Cordeiro, G. M., Alizadeh, M.,Ozel, G.,Hosseini, B., Ortega, E. M. M., and Altun, E. (2017). The generalized odd log-logistic family of distributions: properties, regression models and applications, Journal of Statistical Computation \& Simulation, 87(5), 908-932.

7. Cordeiro, G. M., Alizadeh,M. Tahir, M. H. Mansoor , M. Bourguignonk, M. and Hamedani, G. G. (2016). The beta odd log-logistic generalized family of distributions. Hacettepe Journal of Mathematics and Statistics, 45 (4), $1175-1202$. 
8. Elgarhy, M., Haq, M. and Ozel, G. A new exponentiated extended family of distributions with Applications.Gazi University Journal of Science 30 (3), 101-115.

9. Elgarhy, M., Hassan, A.S., and Rashed, M. (2016). Garhy-generated family of distributions with application. Mathematical Theory and Modeling, 6(2), 1-15.

10. Eugene, N., Lee, C. and Famoye, F. (2002). The beta-normal distribution and its applications. Communications in Statistics \& Theory and Methods, 31, 497-512.

11. Gomes-Silva, F., Ramos, M.W., Percontini, A., Ven^ancio, R., de Brito, E., Cordeiro, G. M.(2017).The odd Lindley-G family of distributions. Austrian Journal of Statistics, 46, 65-87.

12. Hassan, A. S. and Elgarhy, M. (2016 a). KumaraswamyWeibull-generated family of distributions with applications. Advances and Applications in Statistics, 48, 205-239.

13. Hassan, A. S. and Elgarhy, M. (2016 b). A new family of exponentiatedWeibull-generated distributions. International Journal of Mathematics And its Applications, 4, 135-148

14. Hassan, A. S., Elgarhy, M., and Shakil, M. (2017). Type II half Logistic family of distributions with applications. Pakistan Journal of Statistics \&Operation Research, 13(2), 245-264.

15. Haghbin, H.,Ozel, G.,Alizadeh, M., and Hamedani, G. G. (2017). A new generalized odd log-logistic family of distributions. Communications in Statistics - Theory and Methods, 46 (20), 9897-9920

16. Krishnarani, S. D. (2016). On a Power Transformation of Half-Logistic Distribution,Journal of Probability and Statistics, Volume 2016, Article ID 2084236, 10 pages.

17. Morais A. L. and Barreto-Souza, W. (2011). A compound class of Weibull and power series distributions," Computational Statistics and Data Analysis, 55, no. 3, pp. 1410-1425.

18. Shanker, R. and Mishra, A. (2013). A quasi Lindley distribution. African Journal of Mathematics and Computer Science Research, 6, 64-71.

19. Smith R. L. and Naylor J. C. (1987). A comparison of maximum likelihood and Bayesian estimators for the three-parameter Weibull distribution," Journal of the Royal Statistical Society. Series C. Applied Statistics, vol. 36, no. 3, pp. 358-369.

20. Tahir, M. H., Cordeiro, G. M., Alizadeh, M., Mansoor, M., Zubair, M. and Hamedani, G. G., (2015). The odd generalized exponential family of distributions with applications, Journal of Statistical Distributions and Applications, 2(1): 1-28.

21. Dey, S., Sharma, V. K., \&Mesfioui, M. (2017). A New Extension of Weibull Distribution with Application to Lifetime Data. Annals of Data Science, 4(1), 31-61.

22. El-Bassiouny, A. H., Abdo, N. F., \& Shahen, H. S. (2015). Exponential Lomax distribution. International Journal of Computer Applications, 121(13).

23. Mahdavi, A., \&Kundu, D. (2017). A new method for generating distributions with an application to exponential distribution. Communications in Statistics-Theory and Methods, 46(13), 6543-6557.

24. Mahmoud, M. A. W., \&Alam, F. M. A. (2010). The generalized linear exponential distribution. Statistics \& probability letters, 80(11), 1005-1014.

25. Murthy, D. P., Xie, M., \& Jiang, R. (2004). Weibull models (Vol. 505). John Wiley \& Sons.

26. Sarhan, A. M., Abd, E. B. A., \&Alasbahi, I. A. (2013). Exponentiated generalized linear exponential distribution. Applied Mathematical Modelling, 37(5), 2838-2849. 